\title{
Maternal vaccination to prevent pertussis in infants
}

Pertussis causes an estimated 16 million cases and 200000 deaths globally each year, with most of the deaths occurring in infants younger than 2 months of age. ${ }^{[1]}$ Young infants with pertussis may present with severe disease such as pneumonia, apnoea or seizures, and severe illness is more common in HIV-infected and HIV-exposed children than in HIV-uninfected children. ${ }^{[2]}$ The burden of Bordetella pertussis disease is highest in young infants ( $<3$ months of age) and has almost doubled over the last two decades despite the availability of vaccines. ${ }^{[1]}$ The recent increase in incidence of pertussis infection has been facilitated by an increasing pool of susceptible individuals in the community. As fewer adults (including women of child-bearing age) and children have experienced natural pertussis infection, there is less circulating natural immunity among the adult population, and vaccine-mediated immunity after primary childhood vaccination wanes with time. Furthermore, the duration of protection afforded by the newer acellular pertussis vaccine $(1-3$ years) is shorter than that for the whole-cell vaccine ( $5-10$ years). As primary vaccination schedules against pertussis begin at 6 weeks of age, the young infant is especially vulnerable to pertussis. The primary administration of pertussis vaccine to infants and the vaccination of close contacts to prevent transmission ('cocooning') are measures that will reduce the burden of pertussis, but the protection of young infants from pertussis is most likely achieved through maternal vaccination - a strategy that has been shown as safe and effective. ${ }^{[3]}$

The objective of maternal pertussis vaccination is twofold: it induces or boosts antibody responses in the pregnant woman, which protects the infant through early infancy, and it protects the mother against pertussis infection that may be passed on to her infant postnatally. The success of maternal vaccination in preventing disease in early infancy is dependent on several factors. Firstly, an immunogenic vaccine is required, to produce sufficient protective antibody levels. Secondly, efficient transplacental transfer of maternal IgG antibody needs to occur during the third trimester. Thirdly, the half-life of the transferred antibody should be of sufficient duration in the infant. In addition, the protective epitope-specific IgA antibodies in breastmilk may offer some protection.

When a vaccine is administered to a pregnant woman, the safety and immunogenicity of the vaccine to the mother-fetus dyad are major considerations. Although some safety and immunogenicity trials are yet to be reported, the current consensus of opinion is that maternal immunisation with the Tdap (tetanus, diphtheria and acellular pertussis) vaccine is safe. Indeed, maternal vaccination is now recommended to prevent pertussis infection in vulnerable young infants. In the USA and UK, the immunisation of pregnant women with a Tdap or dTaP/IPV (diphtheria, tetanus, acellular pertussis and inactivated poliomyelitis) vaccine has been standard practice since 2012. These recommendations are based primarily on observational and case-control studies that demonstrated the effectiveness of a five-component acellular-pertussis-containing vaccine $^{[4,5]}$ Although vaccine efficacy is usually demonstrated by controlled double-blind randomised clinical trials, these trials are expensive to perform and often require a very large number of study participants. Nonetheless, the effectiveness of the dTaP/IPV vaccine in preventing pertussis in infancy was greater than $90 \%$ even though serological correlates of protection have not been determined. Even though lower pertussis antibody responses after primary vaccination are noted in infants born to mothers who received Tdap vaccine, maternal pertussis immunisation is more beneficial, and recommended for the prevention of severe disease in young infants.

The optimal time to administer Tdap vaccine to the mother is between 27 and 36 weeks of gestation - vaccination during this period induces maximal antibody responses in the mother, so that adequate amounts of IgG1 are transferred to the fetus. Maternal antibody transferred to the young infant has an antibody half-life that provides adequate protection during the first 2 months of life, when the infant is especially vulnerable to pertussis infection. Unlike maternal influenza vaccination, where early vaccination during pregnancy favourably alters maternal and fetal health outcomes, pertussis vaccination can be delayed to the third trimester because the adequate production of protective Tdap antibodies occurs about 2 weeks after vaccination. If the mother is vaccinated before conception of a planned pregnancy, the levels of maternal antibody will decline during the pregnancy and inadequate levels of protective antibody will be transferred to the fetus. Thus, vaccinating the mother during preconception care or immediately after the birth of the newborn will not protect the young infant against pertussis.

\section{South African situation}

Maternal vaccination is emerging as a favoured strategy to reduce the burden of neonatal deaths globally. In South Africa (SA), the only form of maternal immunisation presently recommended is vaccination with tetanus toxoid to prevent neonatal tetanus infection. Pertussis infection is common in SA infants, ${ }^{[2]}$ and further studies to determine the efficacy or effectiveness of the acellular pertussis vaccine will almost certainly be needed. In particular, determination of the safety and immunogenicity of vaccination in HIV-infected pregnant women is required. Nonetheless, the introduction of pertussis vaccination into the public maternal immunisation programme is likely to reduce the burden of pertussis disease in early infancy.

\section{Ziyaad Dangor \\ Sanjay G Lala}

Department of Paediatrics and Child Health, School of Clinical Medicine, Faculty of Health Sciences, University of the Witwatersrand, Johannesburg, South Africa

\section{Corresponding author: Z Dangor (ziyaad.dangor@wits.ac.za)}

1. Centers for Disease Control and Prevention. Pertussis (Whooping Cough) September 2016. http://www.cdc.gov/pertussis/index.html (accessed 9 September 2016)

2. Muloiwa R, Dube FS, Nicol MP, Zar HJ, Hussey GD. Incidence and diagnosis of pertussis in South African children hospitalized with lower respiratory tract infection. Ped Infect Dis J 2016;35(6):611-616. DOI:10.1097/ INF.0000000000001132

3. Lindsey B, Kampmann B, Jones C. Maternal immunization as a strategy to decrease susceptibility to infection in newborn infants. Curr Opin Infect Dis 2013;26(3):248-253. DOI:10.1097/QCO.0b013e3283607a58

4. Amirthalingam G, Andrews N, Campbell $\mathrm{H}$, et al. Effectiveness of maternal pertussis vaccination in England: An observational study. Lancet 2014;384(9953):1521-1528. DOI:10.1016/S0140-6736(14)60686-3

5. Dabrera G, Amirthalingam G, Andrews N, et al. A case-control study to estimate the effectiveness of maternal pertussis vaccination in protecting newborn infants in England and Wales, 2012-2013. Clin Infec Dis 2015;60(3):333-337. DOI:10.1093/cid/ciu821

S Afr J Child Health 2016;10(3):146. DOI:10.7196/SAJCH.2016.v10i3.1272 